\title{
MENINGOENCEFALOCELE TRANSESFENOIDAL TRANSPALATINA
}

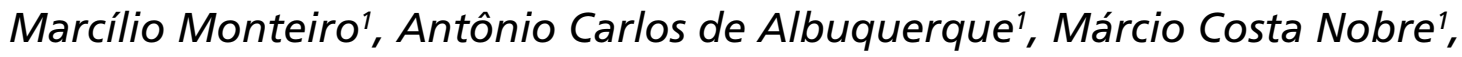 \\ Adriano Teixeira Veloso', Vandete Aguiar Mendes ${ }^{1}$, Lucídio Duarte de Souza Filho², \\ Marcelo José da Silva², Geraldo Vítor Cardoso Bicalho
}

\begin{abstract}
RESUMO - A meningoencefalocele transesfenoidal é rara apresentação dos disrafismos do tubo neural. Caracteriza-se pela herniação do tecido neural através de uma falha óssea no osso esfenóide. A apresentação clínica é variável, podendo ser desde assintomática até obstrução das vias aéreas, rinorréia, meningite, disfunção hipotalâmica e anomalias ópticas. Seu tratamento cirúrgico é controverso. Descrevemos o caso de um menino de 7 anos que apresentava uma estrutura pulsátil preenchendo o teto da cavidade oral, disgenesia palatina e hipertelorismo. 0 diagnóstico de meningoencefalocele transesfenoidal transpalatina foi confirmado pela tomografia computadorizada e ressonância magnética. A criança foi operada com sucesso pela via transpalatal/transesfenoidal.
\end{abstract}

PALAVRAS-CHAVE: meningoencefalocele transesfenoidal transpalatina, encefalocele, acesso transpalatal/transesfenoidal.

\section{Transspheinoidal transpalatal meningoencephalocele}

ABSTRACT - Transspheinoidal meningoencephalocele is a rare presentation of dysraphism of the neural tube. It is characterized by the herniation of the neural tissue through a bony defect in the sphenoid bone. The clinical presentation is variable. It may be assymptomatic or it may include an upper airway obstruction, rhinomhea, meningitis, hypothalamic dysfunction and optic anomalies. The surgical treatment is controversial. We describe the case of a 7-year-old boy who presented a pulsate structure filling the palate, palate digenesis and hypertelorism. The diagnosis of transsphenoidal transpalatal meningoencephalocele was confirmed by a computerized tomography and a magnetic resonance imaging. The child was operated on by the transpalatal/transspheinoidal approach with a good result.

KEY WORDS: transsphenoidal transpalatal meningoencephalocele, encephalocele, transpalatal/transspheinoidal approach.

Encefalocele basal é uma rara entidade patológica que ocorre em 1:35000 nascimentos ${ }^{1-6}$. Constitui 1 a $10 \%$ de todas as encefaloceles ${ }^{3}$. Caracteriza-se pela herniação do tecido neural através de um defeito nas estruturas ósseas da base do crânio ${ }^{2,7,8}$. A encefalocele basal é classificada em transetmoidal, esfenoetmoidal, esfenoorbital, esfenomaxilar e transesfenoidal ${ }^{8,9} .0$ tipo transesfenoidal é o menos freqüente, representando $5 \%$ das encefaloceles da base do crânio ${ }^{2,7,10}$.

Relataremos um caso de meningoencefalocele transesfenoidal transpalatina diagnosticado e tratado em nosso serviço.

\section{CASO}

Menino, leucoderma de 7 anos de idade, atendido na Santa Casa de Montes Claros-MG com relato de déficit de aprendizagem, dificuldade para alimentar, obstrução nasal, respiração bucal e voz anserina. $\mathrm{Na}$ admissão, o exame físico revelou a presença de estrutura ovalada, de coloração rósea, pulsátil, preenchendo o teto da cavidade oral e disgenesia palatal. A criança apresentava hipertelorismo e leve atraso no desenvolvimento neuropsicológico, sem outras anormalidades evidentes. As avaliações endocrinológica e oftalmológica foram normais. A tomografia de crânio (TC) e a ressonância magnética (RM) evidenciaram falha óssea esfenopalatina com presença de meningoencefalocele transesfenoidal transpalatina, hipófise em projeção aparentemente tópica, agenesia do corpo caloso, paquigiria e dilatação assimétrica dos ventrículos laterais (Fig 1). A arteriografia dos vasos cerebrais foi normal.

Cinco meses após o atendimento inicial, o paciente foi submetido a correção cirúrgica da malformação pela via transpalatal/transesfenoidal. Sob anestesia geral, realizouse craniotomia de $1,5 \mathrm{~cm}$ de diâmetro na região parietal posterior direita. Em seguida instalou-se uma derivação ventricular externa (DVE). Procedeu-se dissecção da mucosa e identificação da dura-máter. Dissecou-se minuciosamente

${ }^{1}$ Neurocinurgião do Hospital Santa Casa de Montes Claros MG, Brasil; ${ }^{2}$ Acadêmico do $8^{\circ}$ período do curso de Medicina da Faculdade Estadual de Montes Claros (UNIMONTES), Montes Claros MG, Brasil.

Recebido 18 Novembro 2005, recebido na forma final 13 Março 2006. Aceito 11 Abril 2006.

Dr. Marcílio Monteiro - Rua Aracajú 61 - 39402-267 Montes Claros MG - Brasil. E-mail: trabalhoglm@yahoo.com.br 

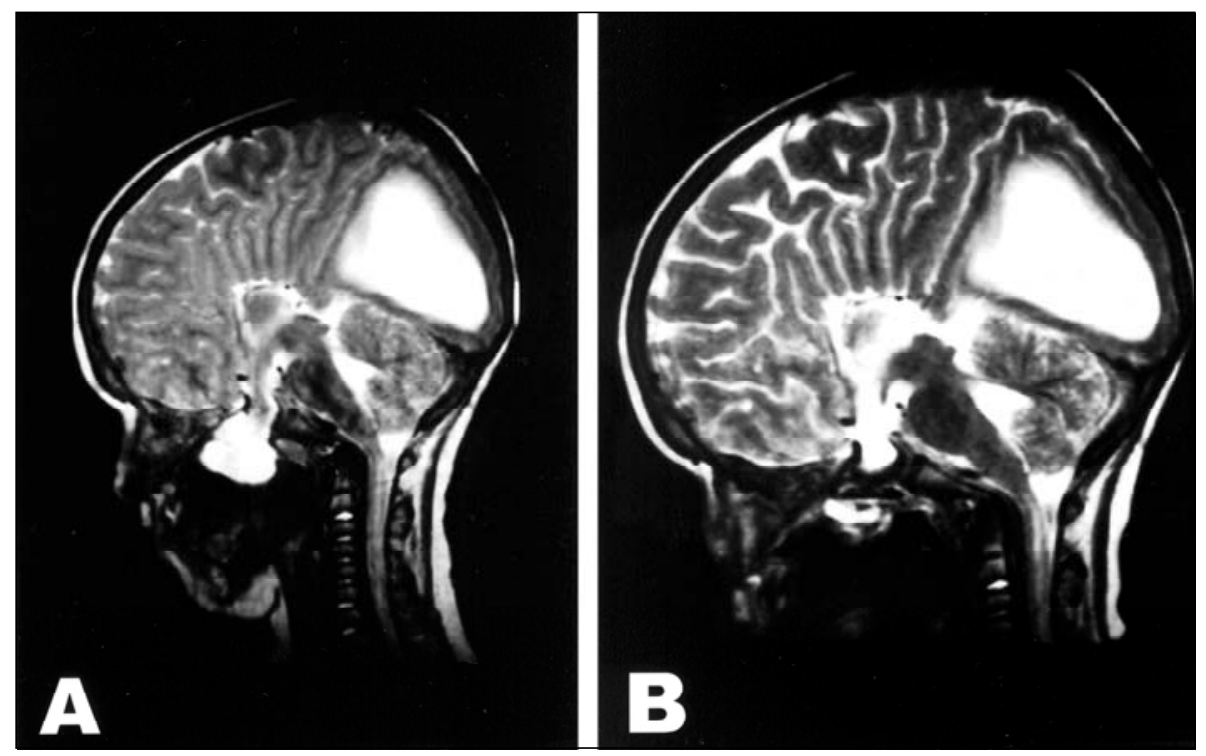

Fig 1. (A) Corte sagital de RM em $T 2$ pré-operatório demostrando a encefalocele transesfenoidal; $(B)$ Corte sagital de RM em $T 2$ pósoperatório mostrando a redução da encefalocele.
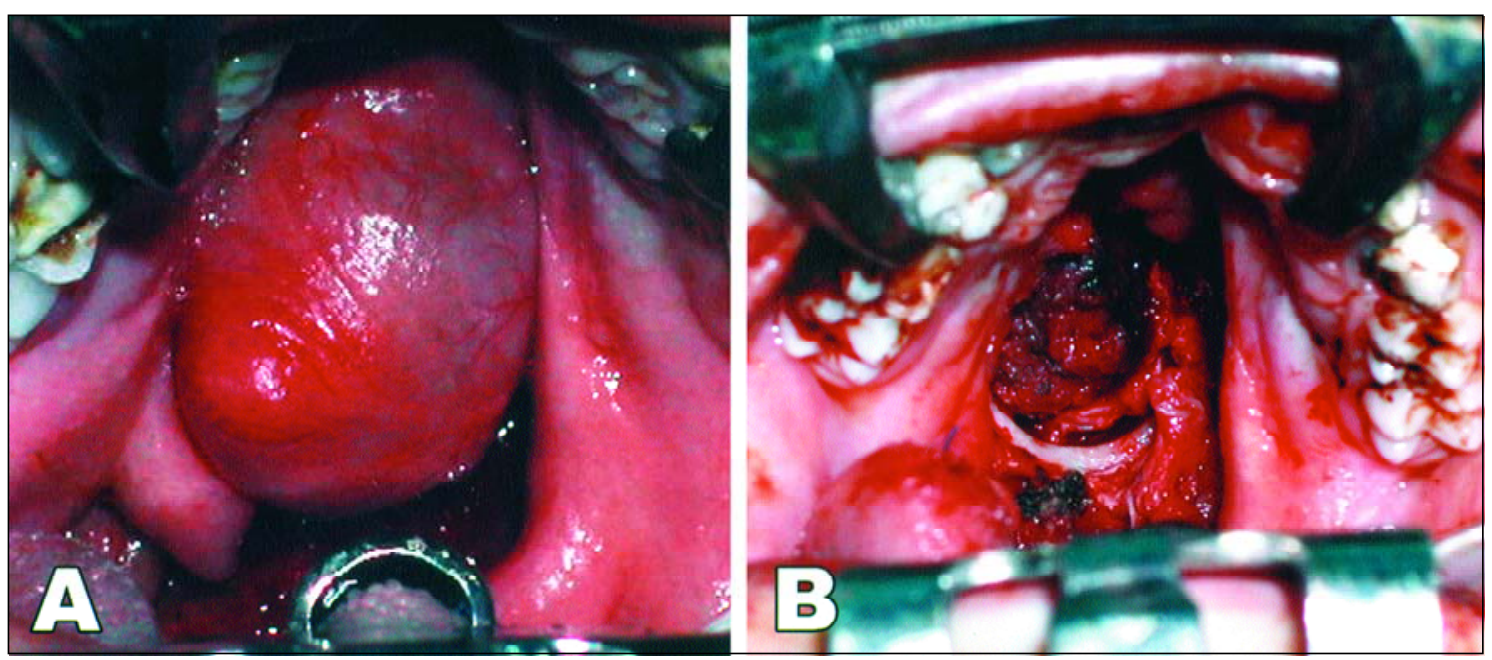

Fig 2. (A) Exposição da encefalocele no pré-operatório; (B) Aspecto da cavidade oral após a dissecção e redução da encefalocele com exposição das bordas do osso esfenóide.

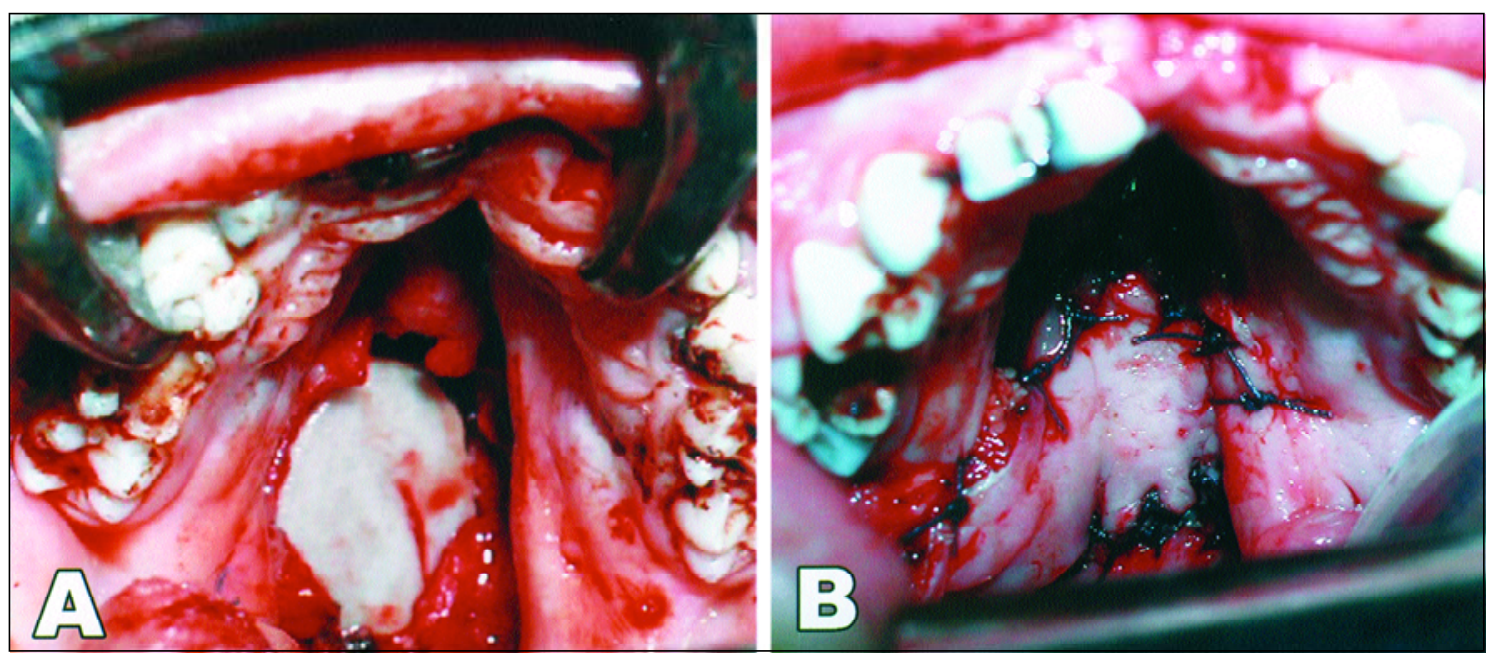

Fig 3. (A) Aspecto do flap ósseo recobrindo falha óssea na base do crânio; (B) Aspecto final após a síntese do retalho de músculo bucinador recobrindo flap ósseo e isolando-o da cavidade oral. 
o saco herniado, reduzindo-o com auxílio de coagulação bipolar, sem abertura da dura-máter (Fig 2). Fez-se a identificação circunferencial das bordas do defeito ósseo e utilizou-se o flap ósseo proveniente da craniectomia para reconstrução da base do crânio (Fig 3). Para o fechamento, foi realizado um enxerto pediculado da mucosa da cavidade oral juntamente com rotação do músculo bucinador. O paciente foi extubado após 48 horas. Retirou-se a DVE no quinto dia após a cirurgia.

A criança apresentou diabetes insipidus ao terceiro dia de pós-operatório. Recebeu alta hospitalar com boas condições clínicas, em uso de desmopressina. A RM de controle foi realizada após 45 dias, evidenciando aspecto pós-operatório satisfatório (Fig 1). Atualmente, o paciente encontrase em controle ambulatorial, mantendo poliúria compensada, sem uso de desmopressina.

Os pais da paciente assinaram o termo de consentimento para publicar o caso.

\section{DISCUSSÃO}

A causa exata da encefalocele basal permanece obscura, embora tenha sido demonstrado haver influência genética na origem de algumas variantes de meningocele em estudos experimentais com exposição à irradiação $X$, doses excessivas de vitamina $A$ e azul de triptano 9 . A teoria clássica atribui a encefalocele transesfenoidal à falta de separação da neurectod e rma da superfície do ectoderma durante a form ação do tubo neural, que dessa forma impede que o tecido do mesoderma se interponha entre as duas camadas germinativas ${ }^{2,8}$. Uma outra teoria considera a existência de um defeito ósseo primário resultante de uma alteração no processo de formação da cartilagem e na ossificação do crânio, ocasionando uma herniação secundária das estruturas encefálicas ${ }^{8}$.

A encefalocele transesfenoidal associa-se com anomalias do desenvolvimento facial, do sistema óptico e do encéfalo ${ }^{1,8,11,12}$. As malformações faciais estão quase sempre presentes e as características encontradas incluem hipertelorismo, fissura nasal mediana, base nasal alargada, fenda labial ou palatal, síndrome da fenda facial medial ou crânio bífido oculto fron$\mathrm{tal}^{9,13,14}$. Anomalias ópticas incluem anoftalmia ou microftalmia, colobomas, anormalidades retinianas e hipoplasia do nervo ou do quiasma ópticos ${ }^{9,15}$. Alterações cerebrais abrangem agenesia do corpo caloso, h i d rocefalia e distopia ou hipoplasia pituitária11,16,17.

A encefalocele transesfenoidal transpalatina geralmente é assintomática ou um achado ocasional, sendo a apresentação mais comum a de uma massa localizada na linha média craniana ${ }^{8}$. As manifestações clínicas, quando presentes, em parte dependem da idade do paciente ${ }^{9}$. Na ausência de alterações faciais, o diagnóstico pode ser retardado para a adolescência ou idade adulta, quando rinorréia, meningite ou defeito visual prog ressivo inexplicável incitam uma avaliação e diagnóstico ${ }^{9,13,17,18}$.Também podem ocorrer hipotireoidismo, déficit de crescimento, hipogonadismo e diabetes insípidus ${ }^{4,9,18}$. Em decorrência do defeito esfenoidal, pode haver herniação das estruturas intracranianas, incluindo o terceiro ventrículo, segmentos do hipotálamo e pituitária, artérias cerebrais anteriores ou quiasma óptico 4,9,13,17. A massa presente no nariz pode ser confundida com um pólipo nasal. A diferença entre o pólipo e a encefalocele é que esta é pulsátil, localiza-se medialmente ao septo nasal e alarga a ponte nasal, enquanto o pólipo não pulsa, localiza-se lateralmente, emana das conchas nasais e não alarga a ponte nasal ${ }^{19}$.

A TC e a RM são necessárias para confirmar o diagnóstico de meningoencefalocele transesfenoidal, para definir a presença de elementos neurais e vasculares na herniação e revelar os defeitos nas estruturas ósseas $^{8,9,20}$. À TC, a encefalocele pode simular uma lesão osteolítica na base do crânio7. A RM é o exame de escolha para o diagnóstico deste tipo de malformação ${ }^{19,21}$. A angiografia digital ou angiorressonância não são rotineiramente necessárias, mas podem ser consideradas se a RM sugerir envolvimento das artérias cerebral anteriores ${ }^{21}$. A TC tridimensional pode ser útil para o planejamento de re $\mathrm{p}$ a roscraniofaciais complexos $^{19,21}$.

Devido à raridade da meningoencefalocele transesfenoidal e ao pequeno número de casos publicados na literatura, a padronização do tratamento é controversa ${ }^{9,13,22}$. A intervenção cirúrgica é indicada quando há obstrução respiratória e rinoliquorréia ou meningite atribuída à lesão ${ }^{20}$. Em indivíduos assintomáticos ou naqueles com alterações hormonais ou déficit visual estável, as indicações para reparo são menos claras $^{9,13,17}$. Alguns autores advogam o tratamento consenador devido à complexa anatomia da lesão e a lenta pro g ressão dos sintomas ${ }^{6,8}$. Essa conduta, entretanto, não é universalmente aceita. Outros autores defendem que o re pa ro, o mais precoce possível, reduz o risco de infecção, o crescimento da encefalocele e o risco de traumatismos no saco herniado ${ }^{9,23}$.

O objetivo do tratamento cirúrgico não é de re staurar todos os elementos neurais da herniação, mas de garantir a desobstrução da nasofaringe, reconstituição da base do crânio e previnir o surgimento de fístula liquórica e meningite?

A eficácia da via transcraniana no tratamento da encefalocele transesfenoidal permanece controversa, 
particularmente em neonatos e crianças ${ }^{20}$. Existem estudos demonstrando que o acesso transcraniano com retração do lobo frontal e elevação dos elementos herniados através da sela turca com ou sem ressecção da cefalocele e seu conteúdo estão associados a alto índice de disfunção hipotalâmica, morbidade e mortalidade ${ }^{13,23}$. Além disso, a utilização dessa via aumenta a complexidade do ato cirúrgico, já que importantes estruturas vasculares e nervosas estão localizadas no local do acesso transcraniano ${ }^{21}$.

A opção pelo tratamento cirúrgico para o paciente aqui relatado foi feita devido à obstrução das vias aé reas e à dificuldade na alimentação ocasionados pelo volumoso saco herniado e também pelo risco de traumatismo dessa estrutura. Utilizou-se a via transpalatal/transesfenoidal por ser tecnicamente mais simples e pela presença de disgenesia palatina, a qual facilita essa via. $O$ acesso transpalatal/transesfenoidal, com ou sem utilização de endoscopia, se mostra seguro e eficaz, mesmo quando não há disgenesia palatina, podendo, neste caso, ser associado à palatotomia ${ }^{19}$. A DVE foi utilizada profilaticamente para prevenir fístulas liqüóricas, sendo instalada no local onde foi removido o flap ósseo utilizado na reconstrução da base do crânio.

A avaliação pré-operatória dessas lesões usando imagens de RM, assim como o acompanhamento de uma equipe multidisciplinar, é essencial para o planejamento e sucesso do tratamento ${ }^{9}$. No caso aqui descrito, apesar de aparentemente a hipófise estar em posição tópica, o paciente apresentou diabetes insipidus provavelmente por manipulação cirúrgica.

Em conclusão, a meningoencefalocele transesfenoidal transpalatina é uma rara anomalia congênita. Apesar de não haver consenso sobre seu tratamento, consideramos a via transpalatal/transesfenoidal uma alternativa válida e segura na terapia desta entidade, particularmente em pacientes sintomáticos.

\section{REFERÊNCIAS}

1. Larsen CE, Hudgins PA, Hunter SB. Skull-base meningoencephalocele p resenting as a unilateral neck mass in a neonate. AJNR 1995;16:11611163.

2. Blustajn J, Netchine I, Frédy D, Bakouche P, Piekarski JD, Meder JF. Dysgenesis of the internal carotid artery associated with transsphenoidal encephalocele: a neural crest syndrome? AJNR 1999;20:1154-1157.

3. Yoshimoto Y, Noguchi M, Tsutsumi Y. A case of transethmoidal encephalocele. No Shinkei Geka 1992;20:249-254 (Abstract in English).

4. Ellyin F, Khatir AH, Singh SP. Hypothalamic-pituitary functions in patients with transsphenoidal encephalocele and midfacial anomalies. J Clin Endocrinol Metab 1980;51:854-856.

5. Kobayashi S, Miyazaki M, Miyagi O, Yodonawa M, Shimizu S. A case of transsphenoidal meningoencephalocele. No Shinkei Geka 1990;18: 1065-1070 ( Abstract in English).

6. JabreA, Tabaddor R, Samaraweera R. Transsphenoidal meningoencephalocele in adults. Surg Neurol 2000;54:183-187.

7. Machado MAC Jr, Barbosa VAO, Pires MCM, et al. Meningoencefaloventriculocele transesfenoidal assintomática em adulto. Arq NeuroPsiquiatr 2001;59:280-282.

8. Mylanus EAM, Marres HAM, Vlietman J, et al. Transalar sphenoidal encephalocele and respiratory distress in a neonate: a case report. Pediatrics 1999;103:1-12.

9. Kennedy EM, Gruber DP, Billmire DA, Crone KR. Transpalatal approach for the extracranial surgical repair of transphenoidal cephaloceles in children. J Neurosurg 1999;87:677-681.

10. Naidich TP, Altman NR, Braffman BH, Mclone DG, Zimmerman RA. Cephaloceles and related malformations. AJNR 1992;13:655-690.

11. Diebler C, Dulac O. Cephaloceles: clinical and neuroradiological appearance. Neuroradiology 1983;25:199-216.

12. Elster AD, Branch CL Jr. Transalar sphenoidal encephaloceles:clinical and radiologic findings. Radiology 1989;170:245-247.

13. Yokota A, Matsukado Y, Fuwa I, et al. Anterior basal encephalocele of the neonatal and infantile period. Neurosurgery 1986;19:468-478.

14. DeMyer $W$. The median cleft face syndrome: differential diagnosis of cranium bifidum occultum, hypertelorism and median cleft nose, lip and palate. Neurology 1967;17:961-971.

15. Goldhammer Y, Smith JL. Optic nerve anomalies in basal encephaloceles. Arch Ophthalmol 1975;93:115-118.

16. Mo o reMH, Lodge ML, David DJ. Basal encephalocele: imaging and exposing the hernia. Br J Plast Surg 1993;46:497-502.

17. Smith DE, Murphy MJ, Hitchon PW, et al. Transsphenoidal encephaloceles. Surg Neurol 1983;20:471-480.

18. Soyer P, Dobbelaere P, Benoit S. Transalar sphenoidal encephalocele: uncommon clinical and radiological findings. Case report. Clin Radiol 1991;43:65-67.

19. McComb JG. Neurological surgery: a comprehensive re fe rence guide to the diagnostic and management of neurosurgical problems, Youmans JR, ed 4. Philadelphia: WB Saunders, 1996:829-841.

20. Tsutsumi K, Asano T, Shigeno T, Matsui T, Ito S, Kaizu H. Transcranial approach for transsphenoidal encephalocele: report two cases. Surg Neurol 1999;51:252-257.

21. Cohen AR. Encephaloceles of the anterior cranial base. In Rengachary S, Wilkins R (eds): Neurosurgical operative atlas. American Association of Neurological Surgeons. Baltimore: Williams and Wilkins, 1992:367-375.

22. Matson DD. Neurosurgery of infancy and childhood, ed 2. Springfield, III: Charles C Thomas, 1969:68.

23. David DJ. Cephaloceles: dassification, phatology and mamanegementa review. J Craniofacial Surg 1993;4:192-201. 